\title{
Review of current classification, molecular alterations, and tyrosine kinase inhibitor therapies in myeloproliferative disorders with hypereosinophilia
}

\author{
This article was published in the following Dove Press journal: \\ Journal of Blood Medicine \\ 8 August 2013 \\ Number of times this article has been viewed
}

\author{
Violaine Havelange ${ }^{1,2}$ \\ Jean-Baptiste Demoulin' \\ 'de Duve Institute, Université \\ catholique de Louvain, Brussels, \\ Belgium; ' 2 Department of Hematology, \\ Cliniques universitaires Saint-Luc, \\ Université catholique de Louvain, \\ Brussels, Belgium
}

\begin{abstract}
Recent advances in our understanding of the molecular mechanisms underlying hypereosinophilia have led to the development of a 'molecular' classification of myeloproliferative disorders with eosinophilia. The revised 2008 World Health Organization classification of myeloid neoplasms included a new category called "myeloid and lymphoid neoplasms with eosinophilia and abnormalities of PDGFRA, PDGFRB or FGFR1." Despite the molecular heterogeneity of PDGFR (platelet-derived growth factor receptor) rearrangements, tyrosine kinase inhibitors at low dose induce rapid and complete hematological remission in the majority of these patients. Other kinase inhibitors are promising. Further discoveries of new molecular alterations will direct the development of new specific inhibitors. In this review, an update of the classifications of myeloproliferative disorders associated with hypereosinophilia is discussed together with open and controversial questions. Molecular mechanisms and promising results of tyrosine kinase inhibitor treatments are reviewed.
\end{abstract}

Keywords: hypereosinophilia, classification, myeloproliferative disorders, molecular alterations, tyrosine kinase inhibitor

\section{Introduction to hypereosinophilia Eosinophil biology}

The normal eosinophil count ranges between $0.05 \times 10^{9} / \mathrm{L}$ and $0.5 \times 10^{9} / \mathrm{L}$ in peripheral blood and between $1 \%$ and $6 \%$ in bone marrow aspiration. Eosinophils originate from $\mathrm{CD} 34+$ hematopoietic precursor cells under the control of transcription factors (eg, erythroid transcription factor [GATA-1], PU.1, CCAAT-enhancer-binding proteins [CEBPs], and signal transducer and activator of transcription 5A [STAT5]) and cytokines. 'Eosinopoietic' cytokines (mainly interleukin [IL]-5, granulocytemacrophage colony-stimulating factor [GM-CSF], and IL-3) are produced by activated $\mathrm{T}$ lymphocytes, mast cells, and stromal cells, and promote proliferation, differentiation, and survival of normal and neoplastic eosinophils via specific cell surface receptors. ${ }^{1,2}$ Only IL-5 is specific for eosinophils, while IL-3 and GM-CSF stimulate other myeloid lineages. Under various conditions, eosinophils can invade tissues or organs. Eosinophils produce a number of active molecules in their granules, such as eosinophil peroxidase (EPX), eosinophil cationic protein, major basic protein (MBP), and various lipid mediators and several cytokines, including transforming growth factor beta (TGF- $\beta$ ). When eosinophils are activated by different stimuli for a long period of time, the release of eosinophil granule proteins can trigger local inflammation
Correspondence: Violaine Havelange de Duve Institute - Médecine expérimentale, Avenue Hippocrate 75, BI.75.10, B-1200, Brussels, Belgium Tel +3227647585

Fax +3227647430

Email violaine.havelange@uclouvain.be 
and alter the microenvironment, resulting in tissue fibrosis, thrombosis, and severe organ damage.

\section{Definitions}

Blood eosinophilia is usually divided into mild $\left(0.5-1.5 \times 10^{9} / \mathrm{L}\right)$, moderate or marked $\left(1.5-5.0 \times 10^{9} / \mathrm{L}\right)$, and severe or massive $\left(>5 \times 10^{9} / \mathrm{L}\right)^{4}$. Until recently, the definition of hypereosinophilic syndrome was based on the three criteria described by Chusid et al in $1975^{5}$ : (1) a persistent absolute blood eosinophil count $>1.5 \times 10^{9} / \mathrm{L}$ for more than 6 months (or death before 6 months associated with signs and symptoms of hypereosinophilic disease); (2) a lack of evidence of parasite, allergy or another known cause of eosinophilia; and (3) signs or symptoms of organ involvement, including hepatosplenomegaly, congestive heart failure, gastrointestinal dysfunction, diffuse or focal nervous system abnormalities, pulmonary fibrosis, fever, weight loss or anemia. A detailed description of organ damage induced by eosinophils was reviewed by Roufosse et al. ${ }^{6} \mathrm{~A}$ major issue is the lack of robust criteria to define hypereosinophilia-organ damage by radiological or histological examination of the affected tissues. ${ }^{8}$

In 2011, the Working Conference on Eosinophil Disorder and Syndromes (2011 Working Conference) updated the definition of eosinophilic disorders. ${ }^{7}$ The expert panel proposed that the term hypereosinophilia should be used for marked and persistent eosinophilia $\left(>1.5 \times 10^{9} / \mathrm{L}\right.$ in at least two measurements with a minimum interval of 4 weeks). ${ }^{7}$ Such a recommendation may be adapted to the urgent need of therapy in patients with hypereosinophilia-related end-organ damage. ${ }^{8}$ Tissue hypereosinophilia was defined by (1) the presence of more than $20 \%$ of eosinophils in bone marrow aspiration, (2) identification of tissue infiltration by eosinophils, or (3) identification of eosinophil granule proteins on biopsy material. However, objective criteria for tissue hypereosinophilia in extramedullary organs are not available. Immunohistochemical markers for eosinophils (eg, EPX, MBP) are not specific and there are no markers for immature or neoplastic eosinophils. ${ }^{8}$ Finally, the experts defined a new category of patients with eosinophil-related organ damage - eosinophil infiltrates with single-organ dysfunction. ${ }^{7}$

\section{Classification of hypereosinophilia}

The 2011 Working Conference's panel of experts determined a new classification of hypereosinophilia with four variants, as well as a classification of hypereosinophilic syndromes with three variants (Tables 1 and 2). ${ }^{7}$ Most hypereosinophilia are secondary or reactive; they are caused by allergic reaction ( $80 \%$ of the cases), helminth infections $(8 \%)$, toxic or allergic drug reactions, atopic disorder, or other rare disorders.
Table I Classification of hypereosinophilia

\begin{tabular}{|c|c|}
\hline Proposed terminology & Pathogenesis/definition \\
\hline $\begin{array}{l}\text { Hereditary (familial) } \\
\text { hypereosinophilia }\end{array}$ & Pathogenesis unknown; familial eosinophilia. \\
\hline $\begin{array}{l}\text { Hypereosinophilia of } \\
\text { undetermined } \\
\text { significance }\end{array}$ & $\begin{array}{l}\text { No underlying cause of hypereosinophilia, } \\
\text { no family history. } \\
\text { No symptom of hypereosinophilia. }\end{array}$ \\
\hline $\begin{array}{l}\text { Primary (clonal/neoplastic) } \\
\text { hypereosinophilia }\end{array}$ & $\begin{array}{l}\text { Underlying stem cell, myeloid or } \\
\text { eosinophilic neoplasm (WHO criteria). }\end{array}$ \\
\hline $\begin{array}{l}\text { Secondary (reactive) } \\
\text { hypereosinophilia }\end{array}$ & $\begin{array}{l}\text { Underlying condition/disease in which } \\
\text { eosinophils are non-clonal cells. } \\
\text { Hypereosinophilia is triggered by } \\
\text { cytokines. }\end{array}$ \\
\hline
\end{tabular}

Note: Adapted from J Allergy Clin Immunol, 130(3), Valent P, Klion AD, Horny HP, et al. Contemporary consensus proposal on criteria and classification of eosinophilic disorders and related syndromes, 607-612.e9. Copyright (2012), with permission from Elsevier. ${ }^{7}$

Abbreviation: WHO, World Health Organization.

Secondary hypereosinophilia are polyclonal processes mediated by 'eosinopoietic' cytokines that promote proliferation of eosinophils and their precursors. Overproduction of IL-5 by a subtype of CD4 T helper cell (Th2) can be documented in many cases (eg, in allergic and parasitic disorders). However, the classification of hypereosinophilia is more complex. ${ }^{9}$ Hypereosinophilia can be reactive in hematopoietic neoplasms, such as in Hodgkin's lymphoma, T-cell lymphoma, B-lymphoblastic leukemia/lymphoma, or T-lymphoblastic leukemia with molecular alteration such as $\mathrm{t}(5 ; 14)(\mathrm{q} 35 ; \mathrm{q} 32)$ that activates the IL-3 gene. ${ }^{7}$ In these patients, eosinophils are non-neoplastic cells. The lymphoid variant of hypereosinophilic syndrome is a special subgroup of reactive hypereosinophilic syndrome caused by the non-malignant expansion of clonal Th-2 lymphocytes with an aberrant

Table 2 Classification of syndromes and conditions accompanied by hypereosinophilia

\begin{tabular}{ll}
\hline Proposed terminology & Pathogenesis/definition \\
\hline $\begin{array}{l}\text { Idiopathic hypereosinophilic } \\
\text { syndrome }\end{array}$ & $\begin{array}{c}\text { No underlying cause of } \\
\text { hypereosinophilia. }\end{array}$ \\
$\begin{array}{l}\text { Primary (clonal/neoplastic) } \\
\text { hypereosinophilic syndrome }\end{array}$ & $\begin{array}{c}\text { Underlying stem cell, myeloid or } \\
\text { eosinophilic neoplasm (WHO } \\
\text { criteria). }\end{array}$ \\
Secondary (reactive) & $\begin{array}{l}\text { Underlying condition/disease in which } \\
\text { hypereosinophilic } \\
\text { syndrome }\end{array}$ \\
& $\begin{array}{l}\text { Hypereosinophilia is triggered by } \\
\text { cytokines. }\end{array}$ \\
& Subvariant: lymphoid variant \\
hypereosinophilic syndrome \\
(clonal T-cells identified as the only \\
potential cause).
\end{tabular}

Note: Adapted from J Allergy Clin Immunol, I30(3), Valent P, Klion AD, Horny HP, et al. Contemporary consensus proposal on criteria and classification of eosinophilic disorders and related syndromes, 607-6I2.e9. Copyright (20I2), with permission from Elsevier. ${ }^{7}$

Abbreviation: WHO, World Health Organization. 
immunophenotype (mainly CD3-, CD4+) producing IL-5. ${ }^{10}$ Eosinophils are not in the malignant clone but their number increases reactively in response to eosinopoietic cytokines produced by clonal, aberrant $\mathrm{T}$ lymphocytes. This variant has to be differentiated from hematopoietic stem cell disorders in which both the eosinophils and the lymphocytes belong to the neoplastic clone by molecular and cytogenetic studies. Classification of clonal (neoplastic) hypereosinophilia was recently revised by the 2008 World Health Organization (WHO) classification of myeloid neoplasms. ${ }^{11}$

\section{Myeloproliferative disorders with eosinophilia Diagnostics and classification}

In patients with myeloid or stem cell-derived neoplasms, eosinophils usually belong to the malignant clone, although this is difficult to establish in routine tests. Both clonal and non-clonal eosinophils can coexist. There is no robust immunophenotypic marker or combination of markers to detect immature or neoplastic eosinophils available. ${ }^{8}$ Molecular markers associated with cytogenetic abnormalities are highly indicative of clonal hypereosinophilia in myeloid neoplasms and stem cell neoplasms with eosinophilia. The most common fusion genes involve PDGFRA (plateletderived growth factor receptor, alpha polypeptide), PDGFRB (PDGFR, beta polypeptide), FGFRl (fibroblast growth factor receptor 1), ABL1 (c-abl oncogene 1, non-receptor tyrosine kinase), and JAK2 (Janus kinase 2). ${ }^{12}$ The recurrent molecular abnormalities reported in more than five patients are listed in Table 3. ${ }^{8,12-14}$ Many of them can be detected by conventional karyotyping. CHIC2 (cysteine-rich hydrophobic domain 2) deletion associated with the FIPIL1(FIPI like 1 [S. cerevisiae])-PDGFRA fusion gene is only found using fluorescent in situ hybridization (FISH). In all cases, polymerase chain reaction (PCR) allows the confirmation of molecular alterations, but this is usually not necessary.

The revised 2008 WHO classification of myeloid neoplasms introduced molecular markers as disease-related criteria. Two different categories of myeloid neoplasms with eosinophilia are proposed by the 2008 WHO classification: (1) "chronic eosinophilic leukemia [CEL], not otherwise specified [CEL-NOS],"'11 and (2) "the myeloid and lymphoid neoplasms with eosinophilia and abnormalities of PDGFRA, PDGFRB, or FGFR1." 11 The WHO classification specifies that all patients with PDGFRA, PDGFRB or FGFR1 abnormalities need further diagnostic evaluation to get a final diagnosis of myeloproliferative neoplasms (MPN) or another malignancy. ${ }^{11}$ The 2011 Working Conference's panel of experts agreed
Table 3 Recurrent molecular abnormalities detected in myeloproliferative neoplasms with eosinophilia

\begin{tabular}{ll}
\hline Fusion gene/mutation (molecular) & Cytogenetic abnormality \\
\hline PDGFRA & \\
FIPILI-PDGFRA & $\operatorname{del}(4 \mathrm{q} \mid 2)$ \\
PDGFRB & \\
ETV6-PDGFRB & $\mathrm{t}(5 ; \mid 2)(\mathrm{q} 33 ; \mathrm{p} \mid 3)$ \\
CCDC6-PDGFRB & $\mathrm{t}(5 ; \mid 0)(\mathrm{q} 33 ; \mathrm{q} 2 \mid)$ \\
FGFRI & \\
ZMYM2-FGFRI & $\mathrm{t}(8 ; \mid 3)(\mathrm{p}|\mathrm{I} ; \mathrm{q}| 2)$ \\
FGFRI OP2-FGFRI & ins $(\mid 2 ; 8)(\mathrm{p}|\mathrm{I} ; \mathrm{p}| \mathrm{I} 22)$ \\
CEPI IO-FGFRI & $\mathrm{t}(8 ; 9)(\mathrm{p} \mid 2 ; \mathrm{q} 33)$ \\
JAK2 & \\
PCM I-JAK2 & $\mathrm{t}(8 ; 9)(\mathrm{p} 2 \mid ; \mathrm{p} 24)$ \\
ETV6-JAK2 & $\mathrm{t}(9 ; \mid 2)(\mathrm{p} 24 ; \mathrm{p} \mid 3)$ \\
Others & \\
CBFB-MYHII & inv $(\mid 6)$ \\
BCR-ABLI & $\mathrm{t}(9 ; 22)(\mathrm{q} 34 ; \mathrm{q} \mid \mathrm{I})$ \\
Point mutations & - \\
KIT D8I6V & - \\
JAK2 V6I IF & -
\end{tabular}

Note: For an exhaustive list of fusions, see supplemental tables in Medves and Demoulin. ${ }^{12}$

Abbreviations: PDGFR, platelet-derived growth factor receptor; PDGFRA, PDGFR alpha polypeptide; PDGFRB, PDGFR beta polypeptide; FGFRI, Fibroblast growth factor receptor I; FIPILI, FIPI like I (S. cerevisiae); ETV6, ets variant 6; CCDC6, coiled-coil domain containing 6; ZMYM2, zinc finger, MYM-type 2; CEPI I0, centriolar coiled coil protein II 0 kDa; JAK2, Janus kinase 2; PCM I, pericentriolar material I; CBFb, core-binding factor, beta subunit; $B C R$, breakpoint cluster region; $A B L I$, c-abl oncogene I, non-receptor tyrosine kinase.

with the cytogenetic and molecular WHO classification but underlined two weaknesses - the lack of histologic subclassification and the absence of subgroups with more than one driver mutation. ${ }^{7,8}$ The second mutation can be produced by a subclone, or two separate neoplasms can coexist. Furthermore, eosinophilia is of prognostic significance and each subtype diverges regarding disease biology, prognosis, and response to kinase inhibitors. The KIT D816V mutation is associated with clonal hypereosinophilia in advanced systemic mastocytosis (SM) but not in indolent SM. The high level of serum tryptase $(>100 \mathrm{ng} / \mathrm{mL})$ can reveal an indolent SM in cases of MPN-eo or CEL. The WHO classification does not include lymphocytic and familial categories.

A transient solution was proposed by the expert's panel in the 2011 Working Conference. Minimal diagnostic criteria for CEL and acute eosinophilic leukemia (AEL) were established (Table 4). The molecular and cytogenetic defects in the 2008 WHO classification were detailed and a provisional histopathologic classification was proposed (Table 4). ${ }^{7}$ The cytohistomorphological criteria remain the primary criteria and the molecular and cytogenetic markers will be minor diagnostic criteria. For myeloid neoplasms with hypereosinophilia where criteria for CEL or AEL are not fulfilled, 
Table 4 Hematopoietic neoplasms with hypereosinophilia: comparison between WHO-based definitions and a provisional working definition for morphologic disease variants

Molecular classification and recurrent molecular/chromosome defects

I. Myeloid, lymphoid, and hematopoietic stem cell neoplasm with hypereosinophilia and a recurrent somatic gene defect

A. PDGFRA-rearranged neoplasms

B. PDGFRB-rearranged neoplasms

C. FGFRI-rearranged neoplasms

D. Other defects: JAK2 fusion gene, FLT3 fusion gene

2. Eosinophilic leukemia without a recurrent somatic gene defect (listed above)

A. No gene defect and no chromosome defect detectable

B. With a nonspecific chromosome/gene abnormality

3. WHO-defined myeloid neoplasm with hypereosinophilia (MPN-eo)

A. $\mathrm{Ph}+$ chronic myeloid leukemia (CML-eo)

B. JAK2 V6I7F myeloproliferative neoplasms (MPN-eo)

C. KIT D8I6V+ systemic mastocytosis (SM-eo)

D. CBFB-fusion gene-related acute myeloid leukemia (AML-eo)

E. Myelodysplastic syndromes with hypereosinophilia (MDS-eo)

F. Other WHO-defined myeloid neoplasms with hypereosinophilia

Histopathologic classification

A. Acute eosinophilic leukemia: hypereosinophilia and eosinophils $\geq 30 \%$ and myeloblasts $\geq 20 \%$

B. Chronic eosinophilic leukemia: hypereosinophilia and eosinophils $\geq 30 \%$ and myeloblasts $<20 \%$ and no underlying stem cell, myeloid or lymphoid neoplasm found

C. Other myeloid neoplasm or stem cell neoplasm with hypereosinophilia - WHO classification criteria and hypereosinophilia but eosinophils $<30 \%$

Note: Adapted from J Allergy Clin Immunol, 130(3), Valent P, Klion AD, Horny HP, et al. Contemporary consensus proposal on criteria and classification of eosinophilic disorders and related syndromes, 607-6/2.e9. Copyright (2012), with permission from Elsevier.

Abbreviations: WHO, World Health Organization; PDGFR, platelet-derived growth factor receptor; PDGFRA, PDGFR alpha polypeptide; PDGFRB, PDGFR beta polypeptide; FGFRI, Fibroblast growth factor receptor I; MPN, myeloproliferative neoplasms; $\mathrm{CML}$, chronic myeloid leukemia; SM, systemic mastocytosis; $\mathrm{MML}$, acute myeloid leukemias; MDS, myelodysplastic syndromes; JAK, Janus kinase; CBFB, core-binding factor, beta subunit; FLT3, fms-related tyrosine kinase 3.

the WHO criteria will be followed and the appendix '-eo' will be added in the final diagnosis.

\section{Distinct driving molecular alterations}

Almost half of the patients with criteria for hypereosinophilic syndrome in two different studies published in the early 1980s exhibited features of myeloproliferative disorder (eg, bone marrow hypercellularity, cell lineage abnormalities, myelofibrosis, splenomegaly, vitamin B12 level elevation). ${ }^{15,16}$ Data suggested that these patients had an aggressive disease with a poor prognosis and did not respond to treatment with steroids. ${ }^{17}$

\section{Eosinophilia associated with myeloid leukemia}

Clonal eosinophilia can represent an expanded population derived from the malignant clone. The most frequent example is the subgroup of core-binding factor acute myeloid leukemias (AML). The WHO classification listed a category of AML with recurrent genetic abnormalities including two subgroups: AML with inversion of chromosome 16 (inv[16]) (p13.1q22) or translocation $(\mathrm{t})(16 ; 16)(\mathrm{p} 13.1 ; \mathrm{q} 22)$ (CBFBMYH11AML); and AML with t(8;21)(q22;q22) (RUNX1RUNX1T1). ${ }^{11}$ Several morphological features of eosinophils are characteristic of these AML subtypes. ${ }^{9}$ An increased number of abnormal eosinophils with characteristic large, basophilic, and dense granules are typically found in bone marrow aspiration of AML patients with inv(16). There is no obvious arrest in maturation. The eosinophils derive from the leukemic clone and possess the inv(16) rearrangement. ${ }^{18,19}$ $C B F B$ at $16 \mathrm{q} 22$ encodes the $\beta$-subunit of core-binding factor (CBF), whereas MYH11 at 16q13 encodes the smooth muscle myosin heavy chain (SMMHC). ${ }^{19,20}$ The fusion oncoprotein impairs hematopoietic differentiation but is not sufficient to induce AML. Cooperating mutations in $R A S$ or receptor tyrosine kinase (RTK) (such as FLT3 ITD or KIT mutation) that confer a proliferative and/or survival advantage were found in $70 \%$ of the AML patients with inv(16). ${ }^{19}$ One-third of AML patients with $t(8 ; 21)$ have increased eosinophil precursors and blood eosinophilia. In these cases, $t(8 ; 21)$ is detected in eosinophils that are part of the malignant clone. ${ }^{21,22} R U N X 1$ (also known as $A M L 1$ ) at $21 \mathrm{q} 22.12$ encodes a CBF subunit and the RUNX1-RUNX1T1 fusion disrupts the CBF function, leading to the transcriptional repression of RUNX1 target genes. ${ }^{23}$

In rare cases, clonal eosinophilia can be associated with chronic myeloid leukemia (CML), chronic myelomonocytic leukemia, myelodysplastic syndromes (MDS), or other MPNs, MDS/MPN overlap disorders, and a subset of patients with SM.

\section{Eosinophilia with myeloid neoplasms and PDGFRA abnormalities}

The identification of the FIP1L1-PDGFRA rearrangement led to remarkable advances in the understanding and treatment of clonal myeloproliferative eosinophilias. ${ }^{24}$ The disease was named as CEL or myeloproliferative hypereosinophilic syndrome and is now recognized as a subgroup of myeloid neoplasm in the 2008 WHO classification. ${ }^{11}$ The overwhelming majority of patients with PDGFRAassociated myeloid neoplasms are male. Bone marrow biopsy shows a characteristically hypercellular marrow with increased eosinophils and precursors; eosinophil maturation is typically normal. ${ }^{25}$ In peripheral blood however, eosinophils may exhibit a wide spectrum of 
morphological abnormalities, including hyposegmented or hypersegmented nuclei with cytoplasmic vacuoles and small and sparse granules with clear areas of cytoplasm. ${ }^{9,11,25}$ These alterations are not entirely specific. In many cases, a pronounced mastocytosis is present in scattered or loose non-cohesive aggregates. ${ }^{25}$

The FIP1L1-PDGFRA fusion gene is the most frequently recurrent aberration, detected in $5 \%-15 \%$ of all cases with clonal hypereosinophilia. The fusion gene was detected in eosinophils, neutrophils, mast cells, monocytes, and T-cells or B-cells in some patients, suggesting that the rearrangement arises in a pluripotent hematopoietic progenitor cell. ${ }^{26}$ The fusion transcript results from an 800-kilobase internal deletion on band $4 \mathrm{q} 12$ containing the gene CHIC2. ${ }^{24,27}$ The deletion is cryptic - these patients have a normal karyotype. The deletion results in a fusion of the $5^{\prime}$ end of FIP1L1 and the $3^{\prime}$ end of PDGFRA. ${ }^{24}$ The breakpoints are variables in both genes but the fusions are always in frame. ${ }^{24}$ The breakpoints of FIP1L1 are extended on a region of $40 \mathrm{~kb}$. The role of FIP1L1 in clonal eosinophilia is unknown. FIP1L1 encodes for a protein involved in messenger RNA processing. Breakpoints in PDGFRA occur in a small region that always involves exon 12. ${ }^{24,27}$ PDGFRA encodes an RTK, plateletderived growth factor receptor $\alpha$. The deletion removes the autoinhibitory PDGFRA juxtamembrane domain and leads to the constitutive activation of the tyrosine kinase activity. ${ }^{28}$ In addition, the fusion protein is resistant to degradation, in contrast to wild-type receptors. ${ }^{29}$

FIP1L1-PDGFRA is present in the cell line EOL-1, derived from a patient with AEL. ${ }^{30,31}$ Several studies have aimed to reproduce the disease in mice and hematopoietic stem/progenitor cell models. The activated fusion protein was shown to impose eosinophil-lineage commitment in murine hematopoietic progenitor/stem cells in vitro. ${ }^{32}$ However, in human hematopoietic progenitor cells, FIP1L1-PDGFRA induced colony formation in the absence of cytokines but did not only favor eosinophil development. ${ }^{33}$ We recently transduced in vitro human CD34+ cord blood hematopoietic progenitor cells with FIP1L1-PDGFRA and showed that the fusion oncogene can induce cell proliferation in the absence of cytokine and eosinophilia with IL-3 and IL-5. ${ }^{34}$ Interestingly, we found that FIP1L1-PDGFRA activated the transcription factors STAT (eg, STAT5) and nuclear factor (NF)- $\kappa B .{ }^{34}$ The fusion oncoprotein seems to be a major player in the development of eosinophilia. We cannot rule out that secondary mutations may contribute to the physiopathology of the disease, but until now they were not found. Other myeloid cytogenetic alterations, such as loss of the Y chromosome, trisomy 8 , trisomy $15, \operatorname{del}(6 q)$, $\operatorname{del}(20 q)$, and $\mathrm{i}(17 \mathrm{q})$ have been rarely reported in patients with eosinophil neoplasms, supporting the clonal nature of hypereosinophilia. ${ }^{35}$

However, $65 \%-80 \%$ of cases of eosinophilia associated with myeloid neoplasms remain without known underlying genetic aberration. A few case reports described isolated patients with other fusion products of PDGFRA resulting from chromosomal translocations. These rare patients are sensitive to imatinib. Erben et al developed a quantitative reverse transcriptase $\mathrm{PCR}$ to detect overexpression of the 3'-regions of $P D G F R A$ or $P D G F R B$ as a possible indicator of an underlying fusion. ${ }^{36}$ Sequencing of 87 FIP1L1-PDGFRAnegative hypereosinophilic syndrome patients showed several PDGFRA point mutations (R481G, L507P, I562M, H570R, H650Q, N659S, L705P, R748G, and Y849S). ${ }^{37}$ Four of these in vitro mutations induced growth factor-independent cell proliferation and constitutive phosphorylation of PDGFRA and STAT5. ${ }^{37}$ Mice injected with PDGFRA-mutant cells were treated with oral imatinib. The drug significantly decreased leukemic growth and prolonged survival. ${ }^{37}$ Whether patients carrying such mutations can be successfully treated with imatinib remains to be tested.

\section{Eosinophilia with myeloid neoplasms and PDGFRB or FGFRI abnormalities}

The 2008 WHO classification regrouped under one entity "myeloid and lymphoid neoplasms with eosinophilia and abnormalities of PDGFRA, PDGFRB, or FGFR1." Both $P D G F R B$ at $5 \mathrm{q} 33$ and FGFR1 at 8p11 encode RTK and the mechanisms are similar to PDGFRA. PDGFRB fusion gene is an uncommon cause of clonal eosinophilia reported in only isolated individuals. The most common translocation $\mathrm{t}(5 ; 12)$ (q33;p13) involving ETV6 is found in patients with chronic myelomonocytic leukemia. ${ }^{38}$ The extracellular ligand-binding domain of PDGFRB is replaced by the pointed domain of ETV6, which is required for oligomerization and activation of the kinase domain. Remarkably, the fusion protein also retains the PDGFRB transmembrane domain but is localized in the cytosol. We showed that this hydrophobic domain plays an essential role in the fusion active conformation. ${ }^{39}$ As in the case of FIP1L1-PDGFRA, studies have tried to reproduce the disease in mice and hematopoietic stem/progenitor cell models. ETV6-PDGFRB, in the absence of growth factors, stimulates the proliferation of $\mathrm{Ba} / \mathrm{F} 3$ cell and in vivo promotes hematopoietic cell proliferation in mouse transplantation models, leading to a myeloproliferative disease, but without eosinophilia. ${ }^{40}$ We transduced human CD34+ cord blood hematopoietic stem cells with ETV6-PDGFRB 
and showed an increase in proliferation and eosinophil differentiation with eosinopoietic cytokines. ${ }^{34} \mathrm{NF}-\kappa \mathrm{B}$ seems to be an important mediator of the effects of ETV6-PDGFRB on hematopoietic cell growth and differentiation. ${ }^{34}$

FGFR1 fusion genes are also uncommon. Patients present with eosinophilia and hypercellular bone marrow with variable increase in eosinophils. The cell of origin is believed to be a progenitor cell or a T-cell precursor with potential for myeloid differentiation. The biopsy shows T-cell lymphoblastic leukemia/lymphoma or mixed myeloid/T-cell lineage. The course of the disease is usually aggressive. ${ }^{41}$ The translocation results in a chimeric protein with constitutive activation of FGFR1. The most common translocation is $\mathrm{t}(8 ; 13)(\mathrm{p} 11 ; \mathrm{q} 12)$ involving ZNF198 at 13q12. This disease is also known as 8 p11 myeloproliferative syndrome or stem cell leukemia/lymphoma.

\section{CEL-NOS}

The definition of CEL-NOS in the 2008 WHO classification is based on clonal peripheral blood hypereosinophilia in the absence of diagnostic features associated with another myeloproliferative disorder or AML. The malignant nature of the disease should be confirmed by the presence of a clonal genetic abnormality or blast cells. Genetic alterations may include trisomy(8) but not breakpoint cluster region (BCR)-ABL fusion gene, inv(16) or rearrangement of PDGFRA, PDGFRB or $F G F R 1$. In the absence of genetic alteration, the percentage of blast cells should be more than $2 \%$ in the peripheral blood or more than $5 \%$ in bone marrow, but should not reach the threshold of $20 \%$ associated with AML. ${ }^{11}$ Cases of CEL-NOS are extremely rare.

\section{SM with eosinophilia}

The current WHO definition of SM requires the presence of either one major and one minor criterion or three minor criteria. ${ }^{42}$ The major criterion is multifocal dense infiltrate of mast cells in bone marrow or another extracutaneous organ. The minor criteria are (1) $>25 \%$ of mast cells in bone marrow or non-cutaneous tissue biopsy sections with spindle-shaped or atypical morphology; (2) mast cells in the bone marrow, blood, or involved tissue expressing CD25 and/or CD2; (3) detection of a codon $816 \mathrm{c}$-kit point mutation in blood, bone marrow, or involved tissue; and (4) serum tryptase levels persistently elevated at greater than $20 \mathrm{ng} / \mathrm{mL}$. SM are usually separated into disease variants based on the mast cell burden, involvement of non-mast cell lineages, and disease aggressiveness. ${ }^{42}$

Bone marrow examinations are hypercellular with focal dense, paratrabecular aggregates of atypical spindle-shaped mast cells and increased number of eosinophils and lymphocytes. ${ }^{16,42,43}$ Myelofibrosis and osteolytic or osteosclerotic changes are common in advanced disease. Peripheral blood eosinophilia is found in $>50 \%$ of patients with D816V KIT-positive mast cell leukemia. The mutation can be detected in both eosinophils and CD34+ hematopoietic stem cells in $30 \%$ of patients. ${ }^{44}$

\section{Treatments of clonal eosinophilia: the tyrosine kinase inhibitor (TKI) area}

In contrast with classifications, treatment decisions should be based on symptoms and on molecular defects rather than histomorphological criteria alone. This can be difficult as patients with starkly different underlying diseases can present with identical clinical manifestations. A second obstacle is that only one large multicenter retrospective study is available, in addition to small cases series. ${ }^{45,46}$ Treatment of hypereosinophilic syndrome aims to limit organ damage by controlling the eosinophil count. Standard treatments included prednisone, hydroxyurea, and interferon alfa. In 2002, the TKI imatinib revolutionized the treatment and prognosis of patients with hypereosinophilic syndrome and PDGFR alterations. Imatinib is effective in patients with $A B L 1, P D G F R A$ or $P D G F R B$ fusion genes as well as with some KIT mutations, but not in neoplasms with other kinase mutations such as the FGFR1 fusion gene.

\section{First generation of TKIs: imatinib}

The first case of imatinib treatment of hypereosinophilic syndrome was reported in $2001 .{ }^{47}$ The hypothesis was based on the efficacy of imatinib in chronic myeloid leukemia patients and the probable common pathogenesis. After 4 days of imatinib at $100 \mathrm{mg}$, a complete hematological response was observed and peripheral eosinophils disappeared at day 35 . Subsequently, Gleich et al treated five patients suffering from hypereosinophilic syndrome of unknown origin with $100 \mathrm{mg}$ of imatinib mesylate daily. ${ }^{48}$ Four of the patients with normal serum IL-5 showed a complete hematological response. In 2003, Cools et al treated eleven patients with hypereosinophilic syndrome. ${ }^{24}$ Nine of them had a response to imatinib lasting more than 3 months with an eosinophil count that returned to normal. Cools et al discovered the fusion oncoprotein FIP1L1-PDGFRA in five of the patients. Relapse in one patient was associated with the detection of the T674I mutation in PDGFRA that confers resistance to imatinib. ${ }^{24}$ All published case reports of imatinib treatment of patients with FILP1L1-PDGFRA or PDGFRB rearrangements are listed in Table 5. Nearly all patients with FILP1L1-PDGFRA can be 
Table 5 Published reports of imatinib in hypereosinophilic syndrome with PDGFR alteration

\begin{tabular}{|c|c|c|c|}
\hline Author, year & $\begin{array}{l}\text { Number } \\
\text { of patients treated } \\
\text { with imatinib }\end{array}$ & Responses & $\begin{array}{l}\text { FIPILI- } \\
\text { PDGFRA }\end{array}$ \\
\hline Schaller et $\mathrm{al}^{47}$ & 1 & I CR & NA \\
\hline Gleich et al ${ }^{48}$ & 5 & $4 \mathrm{CR}$ & NA \\
\hline Ault et $\mathrm{a}^{87}$ & I & I CR & NA \\
\hline Pardanani et a ${ }^{88}$ & 7 & $3 \mathrm{CR}$, I PR & NA \\
\hline Cortes et $\mathrm{al}^{89}$ & 9 & $4 \mathrm{CR}$ & NA \\
\hline Cools et $\mathrm{al}^{24}$ & 11 & $9 \mathrm{CR}$ & 5 \\
\hline Pardanani et al ${ }^{90}$ & 5 & $3 \mathrm{CR}$ & 3 \\
\hline Klion et $\mathrm{a}^{91}$ & 7 & $7 \mathrm{CR}$ & 7 \\
\hline $\begin{array}{l}\text { Vandenberghe } \\
\text { et } \mathrm{al}^{92}\end{array}$ & 4 & $4 C R$ & 4 \\
\hline Pardanani et al ${ }^{93}$ & 26 & $12 \mathrm{CR}$ & 8 \\
\hline $\begin{array}{l}\text { Roche-Lestienne } \\
\text { et al }{ }^{94}\end{array}$ & 9 & $7 \mathrm{CR}$ & 6 \\
\hline La Starza et $\mathrm{al}^{95}$ & 12 & $9 \mathrm{CR}$ & 7 \\
\hline Jovanovic et al ${ }^{50}$ & 11 & II CR & 11 \\
\hline Baccarani et al60 & 63 & $27 \mathrm{CR}$ & 32 \\
\hline Helbig et a $\left.\right|^{49}$ & 24 & $13 C R$ & 14 \\
\hline Metzgeroth et $\mathrm{a}^{96}$ & 31 & $22 \mathrm{CR}$ & 16 \\
\hline Helbig et al97 & 22 & $22 \mathrm{CR}$ & 22 \\
\hline Helbig et al ${ }^{98}$ & 8 & $4 \mathrm{CR}$ & 0 \\
\hline Arefi et al ${ }^{99}$ & 19 & $15 \mathrm{CR}$ & $\begin{array}{l}8 \\
\text { PDGFRB } \\
\text { alteration }\end{array}$ \\
\hline Apperley et al ${ }^{100}$ & 4 & $4 \mathrm{CR}$ & 4 \\
\hline David et al ${ }^{101}$ & 12 & $10 \mathrm{CR}$ & 12 \\
\hline Arefi et al ${ }^{99}$ & 8 & $\begin{array}{l}7 \mathrm{CR} \\
\text { I NA }\end{array}$ & 7 \\
\hline Metzgeroth et $\mathrm{a}^{96}$ & 5 & $3 \mathrm{CR}$ & 5 \\
\hline
\end{tabular}

Abbreviations: PDGFR, platelet-derived growth factor receptor; PDGFRA, PDGFR alpha polypeptide; PDGFRB, PDGFR beta polypeptide; CR, complete response; PR, partial response; NA, not applicable; FIPILI, FIPI like I (S. cerevisiae).

managed with low-dose imatinib (100 mg daily to as low as $100 \mathrm{mg}$ weekly). ${ }^{49,50}$ Rapid institution of therapy is important to avoid irreversible complications. The response is usually very rapid; the majority of the patients experienced clinical and hematological responses within the first week of therapy and resolution of bone marrow alterations within the first month. ${ }^{46}$ Resistances were very rare and occurred within the first year of diagnosis. In contrast with $B C R-A B L$ domain mutations, which are a common problem in the treatment of CML, only seven cases of acquired resistances due to a point mutation in the PDGFRA kinase domain have been reported so far, with a median time of 5 months of imatinib therapy. ${ }^{24,27,51-56}$

The T674I mutation within the kinase domain of FIP1L1PDGFRA (adenosine-5'-triphosphate [ATP]-binding region) seems to be the most frequent mutation that appears under imatinib treatment and that causes resistance through steric hindrance mechanisms. The isoleucine (Ile) to threonine (Thr) substitution prevents the deep penetration of imatinib into the ATP-binding pocket. The critical hydrogen bond between $\mathrm{Thr}$ and imatinib is lost and imatinib-binding is destabilized in the kinase domain. ${ }^{57}$ Another patient with resistance to imatinib had two mutations, S601P and L629P in FIP1L1-PDGFRA. ${ }^{54} \mathrm{~S} 601 \mathrm{P}$ is located within the nucleotide binding loop and the new conformation of PDGFRA destabilizes the inactive conformation of the kinase domain that is necessary for the binding of imatinib or sorafenib. ${ }^{58}$ Von Bubnoff et al $^{59}$ identified 27 different FIP1L1-PDGFRA kinase domain mutations, including 25 novel variants which attenuated the imatinib, nilotinib or sorafenib response but did not confer complete inhibitor resistance. It seems that a small number of residues are critical to the interference with binding and inhibition done by PDGFR kinase inhibitors. Of note, in vitro and in vivo findings suggest that imatinib may be effective in patients with activating PDGFRA point mutations. ${ }^{37}$ Imatinib does not seem to be curative in patients with FIP1L1-PDGFRA as the fusion transcript became rapidly detectable after stopping imatinib. ${ }^{60,61}$ Reinitiation of imatinib led to molecular remission. When resistance occurs or side effects do not allow for use of imatinib, another tyrosine kinase inhibitor may be effective. Allogeneic stem cell transplantation was also successfully used in FIP1L1-PDGFRA-positive patients but has to be restricted for patients unresponsive or intolerant to TKIs. ${ }^{62}$

\section{Second generation of TKIs: nilotinib and dasatinib}

Nilotinib can be efficient on the FIP1L1-PDGFRA fusion gene. In vitro, in the EOL-1 cell line, nilotinib was as potent as imatinib in inducing apoptosis and inhibiting proliferation. ${ }^{63}$ Both drugs inhibit the phosphorylation of the PDGFRA tyrosine kinase. In a xenograft model of CEL, complete remission was obtained after 1 week of therapy with both imatinib and nilotinib. ${ }^{64}$ Treatment of two patients resistant to imatinib with nilotinib was successful. ${ }^{55,65}$ Another patient intolerant to imatinib responded to nilotinib and dasatinib. ${ }^{66,67}$ The sensitivity of the T674I mutation to second generation TKIs has been a matter of debate. Von Bubnoff et al reported that nilotinib suppresses the growth of $\mathrm{Ba} / \mathrm{F} 3$ cells transfected with the T674I FIP1L1-PDGFRA mutant. ${ }^{68}$ However, Stover et al reported that nilotinib could not overcome the imatinib resistance conferred by the point mutation T674I in FIP1L1-PDGFRA in the same cellular model, even at high concentrations. ${ }^{69}$ Metzgeroth et al reported a patient with T674I mutation that was insensitive to both nilotinib and sorafenib. ${ }^{51}$ Dasatinib is a dual SRC/ABL1 inhibitor that also inhibits PDGFRs and FIP1L1-PDGFRA fusion but has no effect on imatinibresistant FIP1L1-PDGFRA T674I and D842V mutants. ${ }^{54}$ 


\section{Third generation of TKIs: ponatinib}

Ponatinib has a potent activity towards BCR-ABL1, as well as numerous imatinib-resistant $B C R-A B L 1$ kinase domain mutants, including the T315I mutation..$^{70}$ This third generation TKI was also efficient against the FIP1L1-PDGFRA and FGFR1OP2 (FGFR1 oncogene partner 2)-FGFR1 fusion proteins, as shown in the leukemic EOL and KG1 cell lines. ${ }^{71}$ Ponatinib reduces proliferation, induces apoptosis, and reduces phosphorylation of the FGFR1OP2-FGFR1 fusion protein and substrates in KG1a cell lines. ${ }^{72}$ Importantly, both FIP1L1-PDGFRA T674I and FIP1L1-PDGFRA-D842V mutant kinase were also sensitive to ponatinib. ${ }^{71,73}$ Ponatinib in vitro can also strongly inhibit CUX1 (cut-like homeobox 1)-FGFR1 fusion. ${ }^{73}$ Ren et al recently confirmed that ponatinib can not only inhibit phosphoactivation of six different FGFR1 fusion kinases and their downstream effectors but also inhibit cell growth and clonogenicity of the CD34positive cells transformed by FGFR 1 fusion kinases. ${ }^{74}$ Taken together, these preclinical data point to ponatinib as a very promising therapy for eosinophilic neoplasms associated with RTK mutations. Clinical trials have not yet been reported for this indication.

\section{Other kinase inhibitors}

Sorafenib is a biaryl urea compound with multikinase inhibitory activity. ${ }^{75}$ Sorafenib seems to be an in vitro potent inhibitor for hematological malignancies with FIP1L1-PDGFRA and FIP1L1-PDGFRA T674I mutant. ${ }^{76}$ Lierman et al described a FIP1L1-PDGFRA T674I patient that responded to sorafenib. ${ }^{76}$ However, the clinical response was short because of the emergence of another $\mathrm{D} 842 \mathrm{~V}$ mutation. This mutation is highly resistant to sorafenib, imatinib, and dasatinib. ${ }^{55}$ Sorafenib also failed to block S601P-mutated FIP1L1-PDGFRA. ${ }^{58}$ Structural modeling revealed that the newly identified S601P mutated form of PDGFRA destabilizes the inactive conformation of the kinase domain that is necessary to bind imatinib as well as sorafenib. ${ }^{58}$

Other small molecules have been tested against imatinibresistant FIP1L1-PDGFRA T674I. PKC412 (midostaurin) is an inhibitor of the protein kinase $\mathrm{C}$ family of enzymes. ${ }^{77}$ PKC412 was shown to inhibit FIP1L1-PDGFRA and its T674I mutant in transformed $\mathrm{Ba} / \mathrm{F} 3$ cells (as in murine models) but not the D842V mutant. ${ }^{55,78}$ The novel tyrosine kinase inhibitor EXEL-0862 seems to have an inhibitory activity towards FIP1L1-PDGFRA and even towards the FIP1L1-PDGFRA T674I mutant. ${ }^{75,79}$ Finally, triptolide, a transcription inhibitor, also seems to shut down the expression of FIP1L1-PDGFRA, even with the T674I mutation. ${ }^{80}$
Patients with $P D G F R B$ rearrangement are usually sensitive to imatinib. The imatinib-resistant mutant TEL-PDGFRB T681I was sensitive in vitro and in vivo to nilotinib. ${ }^{69}$ In contrast, patients with JAK2 or FGFR1 abnormalities are less likely to respond to imatinib. Ponatinib showed promising results on FGFR1 fusion kinases. Another interesting drug is TKI258 (dovitinib), which is a RTK inhibitor that increases apoptosis of $\mathrm{Ba} / \mathrm{F} 3$ cells transformed by ZNF198-FGFR1 or FGFR1OP2-FGFR1-positive KG168 ${ }^{81}$

Moreover, imatinib can be efficient in $14 \%-60 \%$ of patients with FIP1L1-PDGFRA-negative hypereosinophilic syndrome. Glucocorticosteroid is the first-line therapy in this group of patients. Eosinophils possess receptors for glucocorticoids which inhibit eosinophil growth and function. The number of glucocorticosteroid receptors detectable in eosinophils correlates with the responses of these cells to glucocorticosteroids. ${ }^{82,83} \mathrm{Via}$ an anti-inflammatory effect, glucocorticosteroids inhibit cytokine-induced expression of adhesion molecules on eosinophils and endothelial cells, and thus eosinophil adhesion and transendothelial migration. If glucocorticosteroid treatment fails, a 1 month dose of standard imatinib (400 mg daily) can be tried. ${ }^{46,84}$ If the patient responds, they probably suffer from a myeloid neoplasm characterized by an unknown mutation sensitive to imatinib.

Is hypereosinophilic syndrome a receptor-tyrosine kinase disease? Remarkably, when a mutated gene is found in patients with a hypereosinophilic syndrome, it is in most cases an RTK such as PDGFRA, PDGFRB or FGFR1, or less frequently KIT or FLT3 ${ }^{14}$ Conversely, mutations and fusions of PDGF receptors have not been associated with other hematological diseases, except in a few isolated case reports, such as the KANK1-PDGFRB that we have described in a thrombocythemia patient. ${ }^{85}$ Non-receptor type tyrosine kinases, such as JAK2 and ABL1, may be associated with hypereosinophilia, but only in rare cases. The reason why these receptors are specifically associated with hypereosinophilia remains unclear. PDGF receptors do not seem to play a major role in normal eosinophil development and may not even be consistently expressed in these cells. ${ }^{34,86}$ We speculate that these receptors may activate a unique set of transcription factors, such as STAT5 and NF- $\kappa B$, which drive eosinophil-differentiation from multipotent progenitors. Our data suggest new opportunities for the treatment of resistant patients.

\section{Conclusion}

Myeloproliferative neoplasms associated with eosinophilia regroup a heterogeneous population of patients with different molecular alterations. The discovery of rearrangements of 
PDGFRA, PDGFRB, and FGFR1 allow for a new molecular classification of these patients. The pathogenesis of PDGFR rearrangement and eosinophilia is still not completely understood. The exquisite response of patients with PDGFRA or PDGFRB rearrangement to imatinib underscores the importance of identifying the underlying molecular alteration. Future challenges remain in testing inhibitors targeting FGFR1 or JAK2 fusion genes or other therapeutic strategies targeting signaling pathways or mechanisms of protein stabilization and degradation.

\section{Acknowlegments}

$\mathrm{VH}$ is a Fellow of the Fonds de la Recherche Scientifique FNRS and the recipient of grants from Plan Cancer (Action 29) and Salus Sanguinis Foundation.

\section{Disclosure}

The authors report no conflicts of interest.

\section{References}

1. Yamaguchi Y, Suda T, Suda J, et al. Purified interleukin 5 supports the terminal differentiation and proliferation of murine eosinophilic precursors. J Exp Med. 1988;167(1):43-56.

2. Tai PC, Spry CJ. The effects of recombinant granulocyte-macrophage colony-stimulating factor (GM-CSF) and interleukin-3 on the secretory capacity of human blood eosinophils. Clin Exp Immunol. 1990;80(3): 426-434.

3. Bach MK, Brashler JR, Stout BK, et al. Activation of human eosinophils by platelet-derived growth factor. Int Arch Allergy Immunol. 1992;97(2):121-129.

4. Rothenberg ME. Eosinophilia. N Engl J Med. 1998;338(22): 1592-1600.

5. Chusid MJ, Dale DC, West BC, Wolff SM. The hypereosinophilic syndrome: analysis of fourteen cases with review of the literature. Medicine (Baltimore). 1975;54(1):1-27.

6. Roufosse FE, Goldman M, Cogan E. Hypereosinophilic syndromes. Orphanet J Rare Dis. 2007;2:37.

7. Valent $\mathrm{P}, \mathrm{Klion} \mathrm{AD}$, Horny HP, et al. Contemporary consensus proposal on criteria and classification of eosinophilic disorders and related syndromes. J Allergy Clin Immunol. 2012;130(3):607-612. e9.

8. Valent P, Horny HP, Bochner BS, Haferlach T, Reiter A. Controversies and open questions in the definitions and classification of the hypereosinophilic syndromes and eosinophilic leukemias. Semin Hematol. 2012;49(2):171-181.

9. Montgomery ND, Dunphy CH, Mooberry M, et al. Diagnostic complexities of eosinophilia. Arch Pathol Lab Med. 2013;137(2):259-269.

10. Roufosse F, Cogan E, Goldman M. Lymphocytic variant hypereosinophilic syndromes. Immunol Allergy Clin North Am. 2007;27(3): 389-413.

11. Vardiman JW, Thiele J, Arber DA, et al. The 2008 revision of the World Health Organization (WHO) classification of myeloid neoplasms and acute leukemia: rationale and important changes. Blood. 2009;114(5): 937-951.

12. Medves S, Demoulin JB. Tyrosine kinase gene fusions in cancer: translating mechanisms into targeted therapies. JCell Mol Med.2012;16(2): 237-248.

13. Valent P, Gleich GJ, Reiter A, et al. Pathogenesis and classification of eosinophil disorders: a review of recent developments in the field. Expert Rev Hematol. 2012;5(2):157-176.
14. Toffalini F, Demoulin JB. New insights into the mechanisms of hematopoietic cell transformation by activated receptor tyrosine kinases. Blood. 2010;116(14):2429-2437.

15. Flaum MA, Schooley RT, Fauci AS, Gralnick HR. A clinicopathologic correlation of the idiopathic hypereosinophilic syndrome. I. Hematologic manifestations. Blood. 1981;58(5):1012-1020.

16. Klion AD. Eosinophilic myeloproliferative disorders. Hematology Am Soc Hematol Educ Program. 2011;2011:257-263.

17. Lefebvre C, Bletry O, Degoulet P, et al. [Prognostic factors of hypereosinophilic syndrome. Study of 40 cases]. Ann Med Interne (Paris). 1989;140(4):253-257. French. [with English abstract].

18. Haferlach T, Winkemann M, Löffler H, et al. The abnormal eosinophils are part of the leukemic cell population in acute myelomonocytic leukemia with abnormal eosinophils (AML M4Eo) and carry the pericentric inversion 16: a combination of May-Grünwald-Giemsa staining and fluorescence in situ hybridization. Blood. 1996;87(6):2459-2463.

19. Reilly JT. Pathogenesis of acute myeloid leukaemia and inv(16) (p13;q22): a paradigm for understanding leukaemogenesis? $\mathrm{Br} \mathrm{J}$ Haematol. 2005;128(1):18-34.

20. Liu P, Tarlé SA, Hajra A, et al. Fusion between transcription factor CBF beta/PEBP2 beta and a myosin heavy chain in acute myeloid leukemia. Science. 1993;261(5124):1041-1044.

21. Kaneko Y, Kimpara H, Kawai S, Fujimoto T. 8;21 chromosome translocation in eosinophilic leukemia. Cancer Genet Cytogenet. 1983;9(2): 181-183.

22. Ishibashi T, Kimura H, Abe R, Matsuda S, Uchida T, Kariyone S. Involvement of eosinophils in leukemia: cytogenetic study of eosinophilic colonies from acute myelogenous leukemia associated with translocation (8;21). Cancer Genet Cytogenet. 1986;22(3):189-194.

23. Meyers S, Lenny N, Hiebert SW. The $t(8 ; 21)$ fusion protein interferes with AML-1B-dependent transcriptional activation. Mol Cell Biol. 1995;15(4):1974-1982.

24. Cools J, DeAngelo DJ, Gotlib J, et al. A tyrosine kinase created by fusion of the PDGFRA and FIP1L1 genes as a therapeutic target of imatinib in idiopathic hypereosinophilic syndrome. $N$ Engl $J$ Med. 2003;348(13):1201-1214.

25. Noel P. Eosinophilic myeloid disorders. Semin Hematol. 2012;49(2): $120-127$.

26. Robyn J, Lemery S, McCoy JP, et al. Multilineage involvement of the fusion gene in patients with FIP1L1/PDGFRA-positive hypereosinophilic syndrome. Br J Haematol. 2006;132(3):286-292.

27. Gotlib J, Cools J. Five years since the discovery of FIP1L1-PDGFRA: what we have learned about the fusion and other molecularly defined eosinophilias. Leukemia. 2008;22(11):1999-2010.

28. Stover EH, Chen J, Folens C, et al. Activation of FIP1L1-PDGFRalpha requires disruption of the juxtamembrane domain of PDGFRalpha and is FIP1L1-independent. Proc Natl Acad Sci U SA. 2006;103(21): 8078-8083.

29. Toffalini F, Kallin A, Vandenberghe P, et al. The fusion proteins TEL-PDGFRbeta and FIP1L1-PDGFRalpha escape ubiquitination and degradation. Haematologica. 2009;94(8):1085-1093.

30. Griffin JH, Leung J, Bruner RJ, Caligiuri MA, Briesewitz R. Discovery of a fusion kinase in EOL-1 cells and idiopathic hypereosinophilic syndrome. Proc Natl Acad Sci U S A. 2003;100(13):7830-7835.

31. Cools J, Quentmeier H, Huntly BJ, et al. The EOL-1 cell line as an in vitro model for the study of FIP1L1-PDGFRA-positive chronic eosinophilic leukemia. Blood. 2004;103(7):2802-2805.

32. Fukushima K, Matsumura I, Ezoe S, et al. FIP1L1-PDGFRalpha imposes eosinophil lineage commitment on hematopoietic stem/ progenitor cells. J Biol Chem. 2009;284(12):7719-7732.

33. Buitenhuis M, Verhagen LP, Cools J, Coffer PJ. Molecular mechanisms underlying FIP1L1-PDGFRA-mediated myeloproliferation. Cancer Res. 2007;67(8):3759-3766.

34. Montano-Almendras CP, Essaghir A, Schoemans H, et al. ETV6PDGFRB and FIP1L1-PDGFRA stimulate human hematopoietic progenitor cell proliferation and differentiation into eosinophils: the role of nuclear factor-אB. Haematologica. 2012;97(7):1064-1072. 
35. Tefferi A, Patnaik MM, Pardanani A. Eosinophilia: secondary, clonal and idiopathic. Br J Haematol. 2006;133(5):468-492.

36. Erben P, Gosenca D, Müller MC, et al. Screening for diverse PDGFRA or PDGFRB fusion genes is facilitated by generic quantitative reverse transcriptase polymerase chain reaction analysis. Haematologica. 2010;95(5):738-744.

37. Elling C, Erben P, Walz C, et al. Novel imatinib-sensitive PDGFRAactivating point mutations in hypereosinophilic syndrome induce growth factor independence and leukemia-like disease. Blood. 2011;117(10): 2935-2943.

38. Golub TR, Barker GF, Lovett M, Gilliland DG. Fusion of PDGF receptor beta to a novel ets-like gene, tel, in chronic myelomonocytic leukemia with $\mathrm{t}(5 ; 12)$ chromosomal translocation. Cell. 1994;77(2):307-316.

39. Toffalini F, Hellberg C, Demoulin JB. Critical role of the plateletderived growth factor receptor (PDGFR) beta transmembrane domain in the TEL-PDGFRbeta cytosolic oncoprotein. J Biol Chem. 2010;285(16): $12268-12278$

40. Cain JA, Xiang Z, O'Neal J, et al. Myeloproliferative disease induced by TEL-PDGFRB displays dynamic range sensitivity to Stat5 gene dosage. Blood. 2007;109(9):3906-3914.

41. Cross NC, Reiter A. Fibroblast growth factor receptor and plateletderived growth factor receptor abnormalities in eosinophilic myeloproliferative disorders. Acta Haematol. 2008;119(4):199-206.

42. Valent P, Akin C, Escribano L, et al. Standards and standardization in mastocytosis: consensus statements on diagnostics, treatment recommendations and response criteria. Eur J Clin Invest. 2007;37(6): 435-453.

43. Horny HP, Parwaresch MR, Lennert K. Bone marrow findings in systemic mastocytosis. Hum Pathol. 1985;16(8):808-814.

44. Garcia-Monter AC, Jara-Acevedo M, Teodosio C, et al. KIT mutation in mast cells and other bone marrow hematopoietic cell lineages in systemic mast cell disorders: a prospective study of the Spanish Network on Mastocytosis (REMA) in a series of 113 patients. Blood. 2006;108(7):2366-2372.

45. Ogbogu PU, Bochner BS, Butterfield JH, et al. Hypereosinophilic syndrome: a multicenter, retrospective analysis of clinical characteristics and response to therapy. J Allergy Clin Immunol. 2009;124(6): 1319-1325. e3.

46. Simon HU, Klion A. Therapeutic approaches to patients with hypereosinophilic syndromes. Semin Hematol. 2012;49(2):160-170.

47. Schaller JL, Burkland GA. Case report: rapid and complete control of idiopathic hypereosinophilia with imatinib mesylate. Med Gen Med. 2001;3(5):9.

48. Gleich GJ, Leiferman KM, Pardanani A, Tefferi A, Butterfield JH. Treatment of hypereosinophilic syndrome with imatinib mesilate. Lancet. 2002;359(9317):1577-1578.

49. Helbig G, Stella-Hołowiecka B, Majewski M, et al. A single weekly dose of imatinib is sufficient to induce and maintain remission of chronic eosinophilic leukaemia in FIP1L1-PDGFRA-expressing patients. $\mathrm{Br} J$ Haematol. 2008;141(2):200-204.

50. Jovanovic JV, Score J, Waghorn K, et al. Low-dose imatinib mesylate leads to rapid induction of major molecular responses and achievement of complete molecular remission in FIP1L1-PDGFRA-positive chronic eosinophilic leukemia. Blood. 2007;109(11):4635-4640.

51. Metzgeroth G, Erben P, Martin H, et al. Limited clinical activity of nilotinib and sorafenib in FIP1L1-PDGFRA positive chronic eosinophilic leukemia with imatinib-resistant T674I mutation. Leukemia. 2012;26(1):162-164.

52. von Bubnoff N, Veach DR, van der Kuip H, et al. A cell-based screen for resistance of Bcr-Abl-positive leukemia identifies the mutation pattern for PD166326, an alternative Abl kinase inhibitor. Blood. 2005;105(4):1652-1659.

53. Ohnishi H, Kandabashi K, Maeda Y, Kawamura M, Watanabe T. Chronic eosinophilic leukaemia with FIP1L1-PDGFRA fusion and T6741 mutation that evolved from Langerhans cell histiocytosis with eosinophilia after chemotherapy. Br J Haematol. 2006;134(5):547-549.
54. Simon D, Salemi S, Yousefi S, Simon HU. Primary resistance to imatinib in Fip1-like 1-platelet-derived growth factor receptor alphapositive eosinophilic leukemia. J Allergy Clin Immunol. 2008;121(4): 1054-1056.

55. Lierman E, Michaux L, Beullens E, et al. FIP1L1-PDGFRalpha D842V, a novel panresistant mutant, emerging after treatment of FIP1L1PDGFRalpha T674I eosinophilic leukemia with single agent sorafenib. Leukemia. 2009;23(5):845-851.

56. Score J, Walz C, Jovanovic JV, et al. Detection and molecular monitoring of FIP1L1-PDGFRA-positive disease by analysis of patient-specific genomic DNA fusion junctions. Leukemia. 2009;23(2):332-339.

57. Gorre ME, Mohammed M, Ellwood K, et al. Clinical resistance to STI571 cancer therapy caused by BCR-ABL gene mutation or amplification. Science. 2001;293(5531):876-880.

58. Salemi S, Yousefi S, Simon D, et al. A novel FIP1L1-PDGFRA mutant destabilizing the inactive conformation of the kinase domain in chronic eosinophilic leukemia/hypereosinophilic syndrome. Allergy. 2009;64(6):913-918.

59. von Bubnoff N, Gorantla SP, Engh RA, et al. The low frequency of clinical resistance to PDGFR inhibitors in myeloid neoplasms with abnormalities of PDGFRA might be related to the limited repertoire of possible PDGFRA kinase domain mutations in vitro. Oncogene. 2011;30(8):933-943.

60. Baccarani M, Cilloni D, Rondoni M, et al. The efficacy of imatinib mesylate in patients with FIP1L1-PDGFRalpha-positive hypereosinophilic syndrome. Results of a multicenter prospective study. Haematologica. 2007;92(9):1173-1179.

61. Klion AD, Robyn J, Maric I, et al. Relapse following discontinuation of imatinib mesylate therapy for FIP1L1/PDGFRA-positive chronic eosinophilic leukemia: implications for optimal dosing. Blood. 2007;110(10):3552-3556.

62. Halaburda K, Prejzner W, Szatkowski D, Limon J, Hellmann A. Allogeneic bone marrow transplantation for hypereosinophilic syndrome: long-term follow-up with eradication of FIP1L1-PDGFRA fusion transcript. Bone Marrow Transplant. 2006;38(4):319-320.

63. Verstovsek S, Giles FJ, Quintás-Cardama A, et al. Activity of AMN107, a novel aminopyrimidine tyrosine kinase inhibitor, against human FIP1L1-PDGFR-alpha-expressing cells. Leuk Res. 2006;30(12): 1499-1505.

64. Wicklein D, Ramos Leal N, Salamon J, et al. Nilotinib and imatinib are comparably effective in reducing growth of human eosinophil leukemia cells in a newly established xenograft model. PLoS One. 2012;7(2):e30567.

65. Tabouret E, Charbonnier A, Mozziconacci MJ, Ivanov V. Low-dose Nilotinib can maintain complete molecular remissions in FIP1L1/ PDGFRA-positive hypereosinophilic syndrome. Leuk Res. 2011; 35(1):136.

66. Ikezoe T, Togitani K, Tasaka T, Nishioka C, Yokoyama A. Successful treatment of imatinib-resistant hypereosinophilic syndrome with nilotinib. Leuk Res. 2010;34(8):e200-e201.

67. Imagawa J, Harada Y, Yoshida T, et al. [Successful treatment with lowdose dasatinib in a patient with chronic eosinophilic leukemia intolerant to imatinib]. Rinsho Ketsueki. 2011;52(7):546-550. Japanese. [with English abstract].

68. von Bubnoff N, Gorantla SP, Thöne S, Peschel C, Duyster J. The FIP1L1-PDGFRA T674I mutation can be inhibited by the tyrosine kinase inhibitor AMN107 (nilotinib). Blood. 2006;107(12):4970-4971; author reply 4972.

69. Stover EH, Chen J, Lee BH, et al. The small molecule tyrosine kinase inhibitor AMN107 inhibits TEL-PDGFRbeta and FIP1L1-PDGFRalpha in vitro and in vivo. Blood. 2005;106(9):3206-3213.

70. O'Hare T, Shakespeare WC, Zhu X, et al. AP24534, a pan-BCR$\mathrm{ABL}$ inhibitor for chronic myeloid leukemia, potently inhibits the T315I mutant and overcomes mutation-based resistance. Cancer Cell. 2009;16(5):401-412. 
71. Gozgit JM, Wong MJ, Wardwell S, et al. Potent activity of ponatinib (AP24534) in models of FLT3-driven acute myeloid leukemia and other hematologic malignancies. Mol Cancer Ther. 2011;10(6): 1028-1035.

72. Chase A, Bryant C, Score J, Cross NC. Ponatinib as targeted therapy for FGFR1 fusions associated with the 8p11 myeloproliferative syndrome. Haematologica. 2013;98(1):103-106.

73. Lierman E, Smits S, Cools J, Dewaele B, Debiec-Rychter M, Vandenberghe $P$. Ponatinib is active against imatinib-resistant mutants of FIP1L1-PDGFRA and KIT, and against FGFR1-derived fusion kinases. Leukemia. 2012;26(7):1693-1695.

74. Ren M, Qin H, Ren R, Cowell JK. Ponatinib suppresses the development of myeloid and lymphoid malignancies associated with FGFR1 abnormalities. Leukemia. 2013;27(1):32-40.

75. Lierman E, Cools J. Recent breakthroughs in the understanding and management of chronic eosinophilic leukemia. Expert Rev Anticancer Ther. 2009;9(9):1295-1304.

76. Lierman E, Folens C, Stover EH, et al. Sorafenib is a potent inhibitor of FIP1L1-PDGFRalpha and the imatinib-resistant FIP1L1-PDGFRalpha T674I mutant. Blood. 2006;108(4):1374-1376.

77. Fabbro D, Ruetz S, Bodis S, et al. PKC412 - a protein kinase inhibitor with a broad therapeutic potential. Anticancer Drug Des. 2000;15(1):17-28

78. Cools J, Stover EH, Boulton CL, et al. PKC412 overcomes resistance to imatinib in a murine model of FIP1L1-PDGFR?-induced myeloproliferative disease. Cancer Cell. 2003;3(5):459-469.

79. Pan J, Quintás-Cardama A, Manshouri T, et al. The novel tyrosine kinase inhibitor EXEL-0862 induces apoptosis in human FIP1L1-PDGFRalpha-expressing cells through caspase-3-mediated cleavage of Mcl-1. Leukemia. 2007;21(7):1395-1404.

80. Jin Y, Chen Q, Lu Z, Chen B, Pan J. Triptolide abrogates oncogene FIP1L1-PDGFRalpha addiction and induces apoptosis in hypereosinophilic syndrome. Cancer Sci. 2009;100(11):2210-2217.

81. Chase A, Grand FH, Cross NC. Activity of TKI 258 against primary cells and cell lines with FGFR1 fusion genes associated with the 8p11 myeloproliferative syndrome. Blood. 2007;110(10):3729-3734.

82. Peterson AP, Altman LC, Hill JS, Gosney K, Kadin ME. Glucocorticoid receptors in normal human eosinophils: comparison with neutrophils. J Allergy Clin Immunol. 1981;68(3):212-217.

83. Prin L, Lefebvre P, Gruart V, et al. Heterogeneity of human eosinophil glucocorticoid receptor expression in hypereosinophilic patients: absence of detectable receptor correlates with resistance to corticotherapy. Clin Exp Immunol. 1989;78(3):383-389.

84. Butterfield JH. Success of short-term, higher-dose imatinib mesylate to induce clinical response in FIP1L1-PDGFRalpha-negative hypereosinophilic syndrome. Leuk Res. 2009;33(8):1127-1129.

85. Medves S, Noël LA, Montano-Almendras CP, et al. Multiple oligomerization domains of KANK1-PDGFR $\beta$ are required for JAK2independent hematopoietic cell proliferation and signaling via STAT5 and ERK. Haematologica. 2011;96(10):1406-1414.

86. Demoulin JB, Montano-Almendras CP. Platelet-derived growth factors and their receptors in normal and malignant hematopoiesis. Am J Blood Res. 2012;2(1):44-56.
87. Ault P, Cortes J, Koller C, Kaled ES, Kantarjian H. Response of idiopathic hypereosinophilic syndrome to treatment with imatinib mesylate. Leuk Res. 2002;26(9):881-884.

88. Pardanani A, Reeder T, Porrata LF, et al. Imatinib therapy for hypereosinophilic syndrome and other eosinophilic disorders. Blood. 2003;101(9):3391-3397.

89. Cortes J, Ault P, Koller C, et al. Efficacy of imatinib mesylate in the treatment of idiopathic hypereosinophilic syndrome. Blood. 2003; 101(12):4714-4716.

90. Pardanani A, Ketterling RP, Brockman SR, et al. CHIC2 deletion, a surrogate for FIP1L1-PDGFRA fusion, occurs in systemic mastocytosis associated with eosinophilia and predicts response to imatinib mesylate therapy. Blood. 2003;102(9):3093-3096.

91. Klion AD, Robyn J, Akin C, et al. Molecular remission and reversal of myelofibrosis in response to imatinib mesylate treatment in patients with the myeloproliferative variant of hypereosinophilic syndrome. Blood. 2004;103(2):473-478.

92. Vandenberghe P, Wlodarska I, Michaux L, et al. Clinical and molecular features of FIP1L1-PDFGRA (+) chronic eosinophilic leukemias Leukemia. 2004;18(4):734-742.

93. Pardanani A, Brockman SR, Paternoster SF, et al. FIP1L1-PDGFRA fusion: prevalence and clinicopathologic correlates in 89 consecutive patients with moderate to severe eosinophilia. Blood. 2004;104(10): 3038-3045.

94. Roche-Lestienne C, Lepers S, Soenen-Cornu V, et al. Molecular characterization of the idiopathic hypereosinophilic syndrome (HES) in 35 French patients with normal conventional cytogenetics. Leukemia. 2005;19(5):792-798.

95. La Starza R, Specchia G, Cuneo A, et al. The hypereosinophilic syndrome: fluorescence in situ hybridization detects the del(4)(q12)FIP1L1/PDGFRA but not genomic rearrangements of other tyrosine kinases. Haematologica. 2005;90(5):596-601.

96. Metzgeroth G, Walz C, Erben P, et al. Safety and efficacy of imatinib in chronic eosinophilic leukaemia and hypereosinophilic syndrome: a phase-II study. Br J Haematol. 2008;143(5):707-715.

97. Helbig G, Moskwa A, Hus M, et al. Durable remission after treatment with very low doses of imatinib for FIP1L1-PDGFR $\alpha$-positive chronic eosinophilic leukaemia. Cancer Chemother Pharmacol. 2011;67(4): 967-969

98. Helbig G, Hus M, Halasz M, et al. Imatinib mesylate may induce longterm clinical response in FIP1L1-PDGFR $\alpha$-negative hypereosinophilic syndrome. Med Oncol. 2012;29(2):1073-1076.

99. Arefi M, García JL, Briz MM, et al. Response to imatinib mesylate in patients with hypereosinophilic syndrome. Int J Hematol. 2012;96(3): 320-326.

100. Apperley JF, Gardembas M, Melo JV, et al. Response to imatinib mesylate in patients with chronic myeloproliferative diseases with rearrangements of the platelet-derived growth factor receptor beta. N Engl J Med. 2002;347(7):481-487.

101. David M, Cross NC, Burgstaller S, et al. Durable responses to imatinib in patients with PDGFRB fusion gene-positive and BCR-ABL-negative chronic myeloproliferative disorders. Blood. 2007;109(1):61-64.
Journal of Blood Medicine

\section{Publish your work in this journal}

The Journal of Blood Medicine is an international, peer-reviewed, open access, online journal publishing laboratory, experimental and clinical aspects of all topics pertaining to blood based medicine including but not limited to: Transfusion Medicine; Blood collection, Donor issues, Transmittable diseases, and Blood banking logistics; Immunohematology; Artificial and alternative blood related medicine; Legal aspects of blood medicine; Historical perspectives. The manuscript management system is completely online and includes a very quick and fair peer-review system. Visit http://www.dovepress.com/ testimonials.php to read real quotes from published authors.

\section{Dovepress}

Submit your manuscript here: http://www.dovepress.com/Journal-of-blood-medicine-journal 